\title{
Varia \\ Climat scolaire et satisfaction professionnelle des enseignant·e.s : un enjeu pour la « qualité du vivre et travailler ensemble n en établissement scolaire
}

\author{
Solange Ciavaldini-Cartaut et Catherine Blaya, Université Côte d'Azur
}

La qualité du vivre et travailler ensemble en milieu scolaire est un enjeu éducatif et de santé au travail pour tous les acteurs et actrices de l'école. Cette recherche menée en France avait pour but de déterminer l'influence du climat scolaire sur la satisfaction professionnelle. Les données ont été recueillis auprès de 50 enseignant.e.s volontaires travaillant dans un collège et un lycée régional. Les résultats mettent en évidence l'impact du climat de cohérence et d'appartenance à une communauté scolaire sur les composantes intrinsèques et extrinsèques de la satisfaction professionnelle. Plusieurs autres facteurs (âge, sexe, expérience, type d'établissement) déterminent un profil d'enseignant.e plutôt insatisfait.e. Pour cet établissement, des préconisations sont faites sur la gouvernance de la direction d'établissement dans une visée de santé au travail.

\section{Introduction}

La " qualité du vivre et travailler ensemble en milieu scolaire " est un enjeu éducatif et de santé pour tous à l'école. En effet, l'établissement scolaire est à la fois un lieu de travail et de vie pour les professionnel.le.s de l'éducation, de l'enseignement et pour les élèves. La visée qui consiste à tendre vers un bon climat scolaire au sein d'un établissement solaire s'inscrit, en France, dans le cadre général de l'accès à l'égalité des chances défendue par la loi du 8 juillet 2013 d'orientation et de programmation pour la refondation de l'école de la République. Toutefois, la qualité de vie en milieu scolaire s'avère problématique au sein des établissements du secondaire en France comme le souligne la littérature institutionnelle (Florin et Guimard, 2017 ; Fotinos et Horenstein, 2011 ; Horenstein, 2006 ; Rascle et Bergugnat, 2016). C'est dans ce contexte que certains établissements scolaires définissent comme prioritaire le maintien d'un bon climat scolaire et d'une qualité de vie perçue comme satisfaisante à la fois par les élèves et les personnels. En lien avec la qualité de vie, nombre de recherches montrent que le climat scolaire influe sur le bien-être, la motivation, la santé et la performance des groupes sociaux à l'école. Parmi les différentes définitions du bien-être au travail, nous retenons celle de Seligman et collègues (2005) : il s'agit d'un état psychologique positif, plaisant et multidimensionnel écartant de fait les aspects négatifs associés aux risques psychosociaux. Avoir la volonté d'améliorer le bien-être et la santé au travail des enseignant.e.s signifie la prise en compte d'une diversité de profils en incluant leur expérience du métier, leur avancée dans la carrière, leur âge, leur sentiment d'appartenance socioprofessionnelle et leurs capacités à s'accommoder aux formes de pénibilité perçue en lien avec leurs conditions de travail (Beaudry et Gagnon, 2019). À cela s’ajoute la satisfaction professionnelle procurée par l'activité individuelle et collective au sein de leur établissement scolaire (Travers et Cooper, 1996).

La perception d'un bon climat scolaire dépend de la satisfaction professionnelle des enseignant.e.s ainsi que de l'efficacité perçue dans l'atteinte des objectifs éducatifs et pédagogiques qu'ils et elles se sont fixés (Sangsue et Vorpe, 2004). Locke (1976) définit la satisfaction professionnelle comme un «état positif ou agréable résultant de l'appréciation par une personne de son travail ou de ses expériences " (p. 130). La qualité des relations interpersonnelles qui se tissent en milieu scolaire se trouve au cœur du climat scolaire perçu et le style de gestion des supérieur.e.s immédiat.e.s l'influence. En outre, ces tensions interpersonnelles peuvent affecter la santé au travail des personnels et susciter un mal-être au travail, un manque d'accomplissement personnel dans l'atteinte des buts fixés (Ciavaldini-Cartaut et al., 2017 ; Travers et Cooper, 1996) une diminution de l'estime de soi (Demirtas, 2010) ou une augmentation de l'épuisement professionnel (Skaalvik et Skaalvik, 2009). La littérature du domaine sur la santé au travail dans les métiers de la relation avec autrui (Lee et al., 2009 ; Travers et Cooper, 1996) explicite deux composantes de la satisfaction professionnelle que sont l'accomplissement personnel d'une part et l'estime de soi et le développement professionnel d'autre part. En atteignant les buts d'accomplissement qu'ils ou elles s'étaient initialement fixés dans leur travail, les professionnel.le.s s'y réalisent et, par-là même, accroissent leur satisfaction professionnelle (Bentea et Anguelache, 2012). Réciproquement, 
une faible satisfaction engendre une forme de détresse psychologique et potentiellement une baisse de l'estime de soi (Demirtas, 2010). Néanmoins, la littérature du domaine demeure partagée à propos de la part respective des composantes de la satisfaction professionnelle (Weiss, 2002) qui affecte le plus l'activité du sujet et sa persistance dans le métier. Il est en effet possible de rencontrer par exemple des enseignant.e.s qui persistent dans le métier bien qu'étant insatisfait.e.s et d'autres qui le quittent malgré l'atteinte des buts qu'ils ou elles s'étaient fixés.

Sur le plan scientifique, peu de recherches francophones documentent les rapports existants entre la perception qu'ont les enseignant.e.s du climat scolaire au sein de leur établissement et leur satisfaction professionnelle et donc peu de pistes sont proposées pour tendre vers une bonne qualité de vie au travail en milieu scolaire (pour une recension Amathieu et Chaliès, 2014). Sur le plan pratique, l'identification de tels rapports nous semble aussi importante au regard du potentiel offert par l'évolution des modalités de gouvernance des directions d'établissement et de l'employeur en général (Hubert, 2015 ; Weissbrodt et al., 2018).

Cette recherche porte sur les facteurs du climat scolaire explicatifs de la satisfaction professionnelle des enseignant.e.s. Sur quels leviers agir pour améliorer le climat scolaire et la satisfaction professionnelle des enseignant.e.s ? Quelles préconisations faire aux personnels de direction pour tendre vers une bonne qualité du vivre et travailler ensemble dans leur établissement ? Afin de répondre à ces questions, le présent article se divise en deux grandes sections. La première précise sur le plan conceptuel le climat scolaire perçu par les enseignant.e.s et les déterminants de leur satisfaction professionnelle. S'ensuit une seconde section où la discussion s'engage autour des enjeux soulevés par l'identification de leurs rapports en matière de préconisations de mode de gouvernance de la direction d'établissement susceptible de prévenir les atteintes à la santé au travail sous la forme du mal-être et d'insatisfaction professionnelle des enseignant.e.s.

\section{Le climat scolaire perçu par les enseignant-e-s}

Il existe plusieurs définitions du climat scolaire qui renvoient à de nombreuses typologies. Pour cet article, nous retiendrons la définition du National School Climate Center de New York (Cohen et al., 2009, cité par Debarbieux, 2015) :

Le climat scolaire renvoie à la qualité et au style de vie à l'école. Le climat scolaire repose sur les modèles qu'ont les personnes de leur expérience de vie à l'école et sur la sociologie compréhensive du sens que les individus donnent à leur vécu. Il reflète les normes, les buts, les valeurs, les relations interpersonnelles, les pratiques d'enseignement, d'apprentissage, de management et la structure organisationnelle inclus dans la vie de l'école. (p. 13)

C'est-à-dire que le "climat scolaire" n'est pas à confondre avec le seul "bien-être" individuel des personnels ou des élèves, mais doit être pensé comme une tension entre des groupes sociaux, qui n’en ont pas forcément la même perception. La mise en tension de ces visions parfois conflictuelles vise à éclairer l'organisation scolaire locale - même dans ses liens avec l'organisation nationale du système scolaire français : ce sont les interactions entre les différents groupes sociaux, leurs accords, leurs conflits, leurs arrangements qui sont en jeu, dans une perspective que la sociologie des organisations peut éclairer (Crozier et Friedberg, 1977), particulièrement dans les difficultés du travail en équipe fortement refusé par une partie des enseignant·ess (Barrère, 2002 ; Tardif et Lessard, 1999).

La typologie du climat scolaire de Debarbieux et al. (2013) qui est retenue dans cet article est proche de celle de Janosz et al. (1998). Elle permet que considérer le climat scolaire comme un phénomène multifactoriel dont les variables constitutives different entre les élèves et les personnels enseignant.e.s et d'éducation (Cohen et al., 2009 ; Mitchell et al., 2010 ; Waasdorp et al., 2011). Le questionnaire proposé est très utilisé en France, notamment maintenant pour les études de la Direction de l'Évaluation, de la Prospective et de la Performance (DEPP) et a reçu le label de la qualité statistique par l'Institut National de la Statistique. Toutefois, la DEPP a jusqu'ici éprouvé de grandes difficultés à faire renseigner les questionnaires par les enseignant.e.s. Certaines de ses questions recoupent amplement celles d'autres questionnaires très employés actuellement sur le sujet, et en particulier celui de l'équipe américano-israélienne de Benbenishity et Astor (2005). Les variables utilisées sont a) le climat d'appartenance et de cohésion au sein de la communauté scolaire (soutien de la direction, mode de gouvernance, travail avec l'équipe éducative) b) le climat pédagogique avec les élèves, c) le climat de sécurité et de respect (justice institutionnelle et organisationnelle, violence perçue), d) et le climat éducatif (respect et cohérence du cadre du vivre ensemble avec les élèves, les parents d'élèves et les pair.e.s enseignant.e.s). Les perceptions du climat scolaire des élèves et des enseignant.e.s n'ont que de 
faibles corrélations entre elles (Brand et al., 2008 ; Gase et al., 2017). Mitchell et al. (2010) montrent que la perception du climat scolaire par les enseignant.e.s est influencée davantage par des facteurs liés à la gestion des comportements en classe alors que les élèves accordent plus d'importance à la relation éducative et pédagogique. Pour Cohen et al. (2009), cette perception est influencée par les relations entre les membres de la communauté éducative, la gouvernance de l'établissement, l'environnement physique et le sentiment d'appartenance à la communauté scolaire. Ainsi, une mauvaise atmosphère de travail prédit un sentiment d'insatisfaction des enseignant.e.s (Travers et Cooper, 1996). Le comportement de la direction de l'établissement, son autorité, l'exemple qu'elle donne et la distance qu'elle observe par rapport aux enseignant.e.s, sont autant de facteurs qui déterminent leur satisfaction et peuvent influencer le climat scolaire perçu.

Il ne s'agit pas de nier les différences qui peuvent exister suivant le contexte social des établissements - qui explique en partie ces différences (par exemple, l'instabilité des équipes est fortement associée aux désavantages sociaux de la population accueillie) (Gottfredson, 2001), mais de mettre en lumière que des interactions propres aux établissements peuvent aussi agir sur la qualité de vie au travail : tout reporter sur les seules inégalités sociales serait induire une sorte de fatalisme et de déterminisme sociologique contre lequel les différentes approches par le « climat scolaire » et - plus techniquement - les modèles multifactoriels tentent de résister.

\section{Les composantes intrinsèques et extrinsèques de la satisfaction professionnelle des enseignant-e-s}

Les travaux scientifiques qui portent sur la satisfaction professionnelle insistent sur le fait que celle-ci dépend à la fois de variables intrinsèques comme l'accomplissement de soi, et de variables professionnelles extrinsèques (l'opportunité d'apprendre de nouvelles choses par la formation, la reconnaissance institutionnelle et sociale du travail fourni, les perspectives d'évolution dans la carrière et donc de développement professionnel) qui varient en fonction d'autres variables, telles que par exemple, l'âge, le sexe, les années d'expérience, le niveau de diplôme.

Selon l'enquête internationale sur l'enseignement et l'apprentissage réalisée en 2013 par l'Organisation de Coopération et de Développement Économiques (OCDE), les enseignant.e.s français·e.s qui déclarent travailler avec des classes regroupant de fortes proportions d'élèves peu performant.e.s ou pertubatrices et perturbateurs ont l'un des niveaux de satisfaction les plus faibles d'Europe. L'insatisfaction peut être suscitée par un manque de confiance en ses aptitudes professionnelles à gérer soit la démotivation des élèves soit leurs comportements perturbateurs ou agressifs (Evers et al., 2004) ce qui va dans le même sens que les résultats de l'étude de Mitchell et al. (2010) sur la perception du climat scolaire. Comme le rapportent également Rascle et Bergugnat (2016) lorsque les enseignant.e.s ont un sentiment de compétence élevé, ils ou elles sont également plus enthousiastes et plus satisfait.e.s de leur travail. C'est alors la composante intrinsèque et les réactions affectives des enseignant.e.s qui sont significatives et qui varient selon chaque professionnel.le (Skaalvik et Skaalvik, 2009). D'après Maroy (2002), parmi les facteurs influençant les composantes extrinsèques de la satisfaction professionnelle, il y a l'isolement au sein de la communauté scolaire (individualisme, mépris, défiance hiérarchique) et le manque de soutien des autres enseignant.e.s ou de la direction d'établissement qui participent d'une mauvaise ambiance de vie et de travail (Evers et al., 2004). Si l'ambiance de vie et de travail se dégrade, l'insatisfaction augmente et la persistance dans le travail diminue.

\section{Mé thodologie}

Pour mesurer l'impact du climat scolaire sur la satisfaction professionnelle des enseignant.e.s, un questionnaire a été renseigné par des personnels volontaires exerçant dans un collège et un lycée régional proches géographiquement. Le lycée régional comprenait une filière d'enseignement général et technologique et une filière du tertiaire dans la voie professionnelle. Le projet de ces deux établissements scolaires était similaire à propos de la qualité de vie et du climat scolaire (2014-2017).

\section{Participant.e.s}

Notre échantillon est composé de 50 enseignant.e.s dont 19 travaillent en collège et 31 en lycée. Nous noterons donc que cet échantillon est relativement réduit et qu'il a par conséquent une portée exploratoire que nous entendons conforter dans nos recherches ultérieures. Les tranches d'âge les plus représentatives sont les 25-45 
ans et les 46-60 ans (Tableau 1).

\section{Tableau 1}

Répartition de l'échantillon ( $N=50)$ en fonction de l'âge et de l'établissement scolaire

\begin{tabular}{llcr}
\hline Établissement & Classe d'âge & Fréquence & $\%$ \\
\hline collège & $1<25$ ans & 1 & 5.263 \\
& $\mathbf{2} 25-45$ ans & $\mathbf{9}$ & $\mathbf{4 7 . 3 6 8}$ \\
& $\mathbf{3} 46-60$ ans & $\mathbf{5}$ & $\mathbf{2 6 . 3 1 6}$ \\
& $4>60$ ans & 4 & 21.053 \\
lycée pro & Total & 19 & 100.000 \\
& $1<25$ ans & 0 & 0.000 \\
& $\mathbf{2} 25-45$ ans & $\mathbf{1 7}$ & $\mathbf{5 4 . 8 3 9}$ \\
& $\mathbf{3} 46-60$ ans & $\mathbf{1 4}$ & $\mathbf{4 5 . 1 6 1}$ \\
& $4>60$ ans & 0 & 0.000 \\
& Total & 31 & 100.000 \\
\hline
\end{tabular}

La première tranche d'âge est composée à 34.61\% d'hommes et à $65.39 \%$ de femmes. La seconde est composée à $26.32 \%$ d'hommes et à $73.68 \%$ de femmes. $77 \%$ des femmes de notre échantillon travaillent en lycée et $47 \%$ en collège. En collège, ce sont les hommes qui sont plus nombreux avec $52 \%$ et $22 \%$ en lycée (Tableau 2).

\section{Tableau 2}

Répartition de l'échantillon en fonction du sexe biologique et de l'établissement (N=50)

\begin{tabular}{llcr}
\hline Établissement & Sexe & Fréquence & $\%$ \\
\hline collège & $1 \mathrm{H}$ & 10 & 52.632 \\
& $2 \mathrm{~F}$ & 9 & 47.368 \\
& Total & 19 & 100.000 \\
lycée & $1 \mathrm{H}$ & 7 & 22.581 \\
& $2 \mathrm{~F}$ & 24 & 77.419 \\
& Total & 31 & 100.000 \\
\hline
\end{tabular}

Ces quatre dernières années, les arrêts maladie déclarés et supérieurs à quatre sont plus nombreux chez les hommes (41\%) que chez les femmes (39\%). De manière générale, ils concernent environ $40 \%$ de notre échantillon (Tableau 3).

\section{Tableau 3}

Nombre d'arrêts maladies cumulés par les enseignant.e.s au cours des quatre dernières années en fonction de leur sexe biologique

\begin{tabular}{llcr}
\hline Sexe & Arrêt maladie $\mathbf{~ 4}$ & Fréquence & \% \\
\hline Hommes & Non & 10 & 58.82 \\
& Oui & 7 & $\mathbf{4 1 . 1 7}$ \\
& Total & 17 & 100 \\
\multirow{3}{*}{ Femmes } & Non & 20 & 60.60 \\
& Oui & 13 & $\mathbf{3 9 . 3 9}$ \\
& Total & 33 & 100 \\
\hline
\end{tabular}

\section{Instrument}

La première section du questionnaire renvoyait aux variables de contrôle soit leur âge autorapporté sur une échelle en quatre points (moins de 25 ans codé 1 ; de 25 à 45 ans codé 2 ; de 46 à 60 ans codé 3 ; plus de 60 ans codé 4) ; leur sexe (codé 1 pour les hommes et 2 pour les femmes); leur établissement d'affectation (codé 1 pour le collège et 2 pour le lycée) ; leur expérience du métier (moins de 3 ans codé 1 ; de 3 à 5 ans codé 2 ; de 6 à 10 ans codé 3 ; plus de 10 ans codé 4) et les arrêts maladie cumulés les quatre dernières années. 
Variable indépendante du climat scolaire :

La seconde section du questionnaire comportait 24 items issus de l'échelle de mesure du climat scolaire et de travail dans l'établissement (Teacher's CEE) retenue dans le rapport de Debarbieux et al. (2013) et l'enquête TALIS (Teaching and Learning International Survey) (OCDE, 2013). Les réponses étaient données sur une échelle de Likert en quatre points allant de 1 (pas du tout d'accord ou mauvaises) à 4 (tout à fait d'accord ou bonnes).

Variable dépendante de la satisfaction professionnelle des enseignant.e.s :

La troisième section du questionnaire comportait 14 items issus de l'échelle de mesure de la satisfaction professionnelle (ST) extraite du rapport sur le climat scolaire de Debarbieux et Fotinos (2010). Les réponses étaient données sur une échelle de Likert en quatre points allant de 1 (pas du tout d'accord) à 4 (tout à fait d'accord).

\section{Résultats}

Résultats de L'analyse factorielle

L'analyse factorielle vise à expliquer le réseau de corrélations parmi un ensemble de variables et se justifie au regard de la taille de notre échantillon et de la nature ordinale (échelles de Likert) des variables utilisées.

Pour l'échelle de mesure du climat scolaire perçu par les enseignant.e.s (Teacher's CEE), l'analyse factorielle réalisée après une réduction des dimensions selon le principe d'un maximum de vraisemblances (compte tenu de la taille de notre échantillon) a regroupé les items sur quatre facteurs (Tableau 4) expliquant $74.95 \%$ de la variabilité de la variable dépendante mesurée par l'échelle. Ces facteurs sont a) le climat d'appartenance à une communauté éducative et scolaire b) le climat sécuritaire c) le climat pédagogique et d) le climat éducatif conformément au modèle de référence de Debarbieux et al. (2013). La matrice des corrélations entre items après une rotation oblique (Pett et al., 2003) et le test de Kaiser-Meyer-Olkin (K.M.O=0.80) permet de vérifier le bon ajustement des items aux facteurs latents. L'alpha de Cronbach de .87 a été calculé à partir d'une matrice de corrélations polychoriques adaptée aux échelles de Likert et documente la fiabilité de l'échelle. Selon la typologie de DeVellis (2016) appliquée aux études exploratoires, un alpha de Cronbach compris entre .60 et .70 est considéré comme un minimum acceptable et entre .80 et .90 comme très bon.

\section{Tableau 4}

Statistiques descriptives de l'échelle Teacher's CEE, moyennes, écarts types et items selon la typologie de Debarbieux et al. (2013)

\begin{tabular}{|c|c|c|c|c|c|c|c|c|c|c|c|c|c|c|}
\hline \multirow[b]{2}{*}{ Items } & \multicolumn{8}{|c|}{$\begin{array}{l}\text { Climat d'appartenance à une communauté } \\
\text { éducative et scolaire } \\
\left(R^{2} \text { ajusté }=44\right) \\
\text { Moyenne }^{\text {oye }}=2.82\end{array}$} & \multicolumn{2}{|c|}{$\begin{array}{c}\text { Climat } \\
\text { sécuritaire } \\
\left(R^{2} \text { ajusté }=.11\right) \\
M^{\text {oyenne }}=3.37\end{array}$} & \multicolumn{2}{|c|}{$\begin{array}{c}\text { Climat } \\
\text { pédagogique } \\
\left(R^{2} \text { ajusté }=.10\right) \\
M^{\text {oyenne }}=2,91\end{array}$} & \multicolumn{2}{|c|}{$\begin{array}{c}\text { Climat } \\
\text { éducatif } \\
\left(R^{2} \text { ajusté }=.8\right) \\
\text { Moyenne }^{\text {oye }}=3.04\end{array}$} \\
\hline & CEE1 & CEE2 & CEE3 & CEE4 & CEE7 & CEE11 & CEE12 & CEE16 & CEE10 & CEE14 & CEE5 & CEE15 & CEE6 & CEE8 \\
\hline$M_{\text {oyenne }}$ & 2.83 & 2.68 & 2.65 & 2.57 & 2.85 & 3.19 & 2.86 & 3.34 & 3.29 & 3.41 & 2.95 & 2.90 & 2.95 & 3.14 \\
\hline Médiane & 3 & 3 & 3 & 3 & 3 & 3 & 3 & 3 & 3 & 3 & 3 & 3 & 3 & 3 \\
\hline Écart Type & 0.77 & 0.82 & 0.75 & 0.88 & 0.65 & 0.72 & 0.86 & 0.72 & 0.69 & 0.64 & 0.49 & 0.65 & 0.42 & 0.54 \\
\hline Mini & 1 & 1 & 1 & 1 & 1 & 1 & 1 & 1 & 2 & 2 & 2 & 2 & 2 & 1 \\
\hline Maxi & 4 & 4 & 4 & 4 & 4 & 4 & 4 & 4 & 4 & 4 & 4 & 4 & 4 & 4 \\
\hline
\end{tabular}

Note : La variable du climat d'appartenance à une communauté scolaire correspond à l'indice global du phénomène étudié à l'issue de l'analyse en composante principale (APC).

Pour l'échelle de mesure de la satisfaction professionnelle des enseignant·e•s (ST), l'analyse factorielle a regroupé les items sur deux facteurs expliquant $66.49 \%$ de la variabilité de la variable dépendante mesurée par l'échelle. Ces facteurs sont a) l'accomplissement personnel et b) la réalisation de soi et développement professionnel. Cette méthode d'extraction a été appliquée dans la mesure où la distribution multivariée des items était normale. La matrice de structure s'est faite à partir de la matrice des corrélations entre items après une rotation oblique. L'indice K.M.O (0.80) confirme un bon ajustement des items aux deux facteurs latents après suppression de cinq items ayant une valeur inférieure à 0.60 . La fiabilité de l'échelle est confirmée par un alpha de Cronbach de .89 (Tableau 5). 


\section{Tableau 5}

Statistiques descriptives de l'échelle de satisfaction professionnelle ST, moyennes, écarts types et composantes selon la typologie de Debarbieux et Fotinos (2010)

\begin{tabular}{|c|c|c|c|c|c|c|c|c|c|}
\hline \multicolumn{5}{|c|}{$\begin{array}{l}\text { Accomplissement personnel } \\
\left(R^{2} \text { ajusté }^{\prime}=.46\right) M^{\text {oyenne }}=2.85\end{array}$} & \multicolumn{5}{|c|}{$\begin{array}{l}\text { Réalisation de soi et } \\
\text { développement professionnel } \\
\left(R^{2} \text { ajusté }=.20\right) \text { Moyenne }=2.54\end{array}$} \\
\hline Item & ST2 & ST5 & ST7 & ST8 & ST11 & ST13 & ST4 & ST10 & ST12 \\
\hline Moyenne & 3.08 & 2.70 & 2.56 & 3.16 & 2.76 & 2.86 & 3.06 & 2.28 & 2.30 \\
\hline Médiane & 3. & 3 & 3 & 3 & 3 & 3 & 3 & 2 & 2 \\
\hline Ecart Type & 0.72 & 1.03 & 1.03 & 0.58 & 0.79 & 0.70 & 0.74 & 0.80 & 0.81 \\
\hline Mini & 1 & 1 & 1 & 2 & 1 & 1 & 1 & 1 & 1 \\
\hline Maxi & 4 & 4 & 4 & 4 & 4 & 4 & 4 & 4 & 4 \\
\hline
\end{tabular}

Note : L'indice global de satisfaction professionnelle est de 2.75 sur une échelle de 4. La dimension de l'accomplissement personnel à l'issue de l'analyse factorielle correspond à l'indice général du phénomène étudié

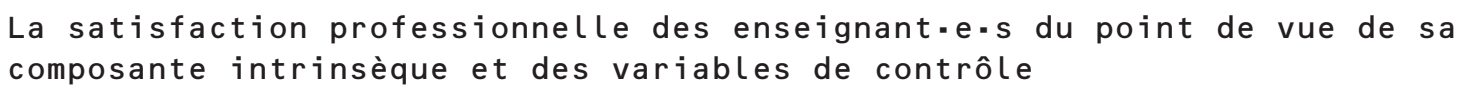

L'indice global de satisfaction professionnelle de notre échantillon est de 2.75 sur 4 . Après avoir vérifié l'homogénéité des variances avec le test de Levene, le test de $T$ Student met en évidence une différence significative entre les femmes et les hommes $(F=4.437 ; p=.04)$ ce qui permet d'affirmer que les hommes $\left(M_{\text {oyenne }}=2.53 ; E T=0.69\right)$ ont en moyenne une satisfaction professionnelle plus faible que les femmes qui composent notre échantillon $\left(M_{\text {oyenne }}=2.78 ; E T=0.46\right)$ (Figure 1$)$.

\section{Figure 1}

Indice global de satisfaction professionnelle en fonction du sexe biologique des enseignant.e.s

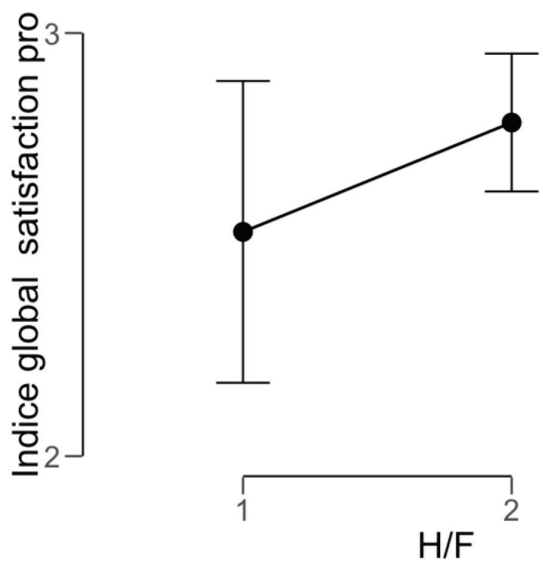

Nous avons voulu savoir si les années d'expérience du métier dans l'établissement scolaire influençaient aussi la satisfaction professionnelle des enseignant.e.s. Comme l'illustre la figure 2, les premières années passées dans l'établissement scolaire affectent plus l'accomplissement personnel (donc la composante intrinsèque de la satisfaction professionnelle) que la réalisation de soi en tant qu'enseignant.e. La réalisation de soi renvoie à la reconnaissance statutaire et au développement professionnel soutenu par les obligations de formation continue des titulaires pendant trois ans (période comprise entre 1 et 3 ans, codé 1 dans la figure 2). Puis, les deux composantes de la satisfaction professionnelle chutent entre 3 et 5 ans d'expérience (période codée 2) ce qui correspond dans la littérature du domaine à l'entrée effective dans le métier où apparaît un essoufflement progressif des ressources acquises lors des formations professionnelles initiales et continues obligatoires (Fotinos et Horenstein, 2011). Ce n'est qu'au cours de la période de maturation de l'expérience du métier que la tendance s'inverse (de 6 à 10 ans codé 3 ; plus de 10 ans codé 4) comme le montrent par ailleurs les résultats de plusieurs études (Demirtas, 2010 ; Karsli et Iskender, 2009). 
Figure 2

Évolution de la composante intrinsèque de la satisfaction professionnelle en fonction des années d'expérience des enseignant.e.s

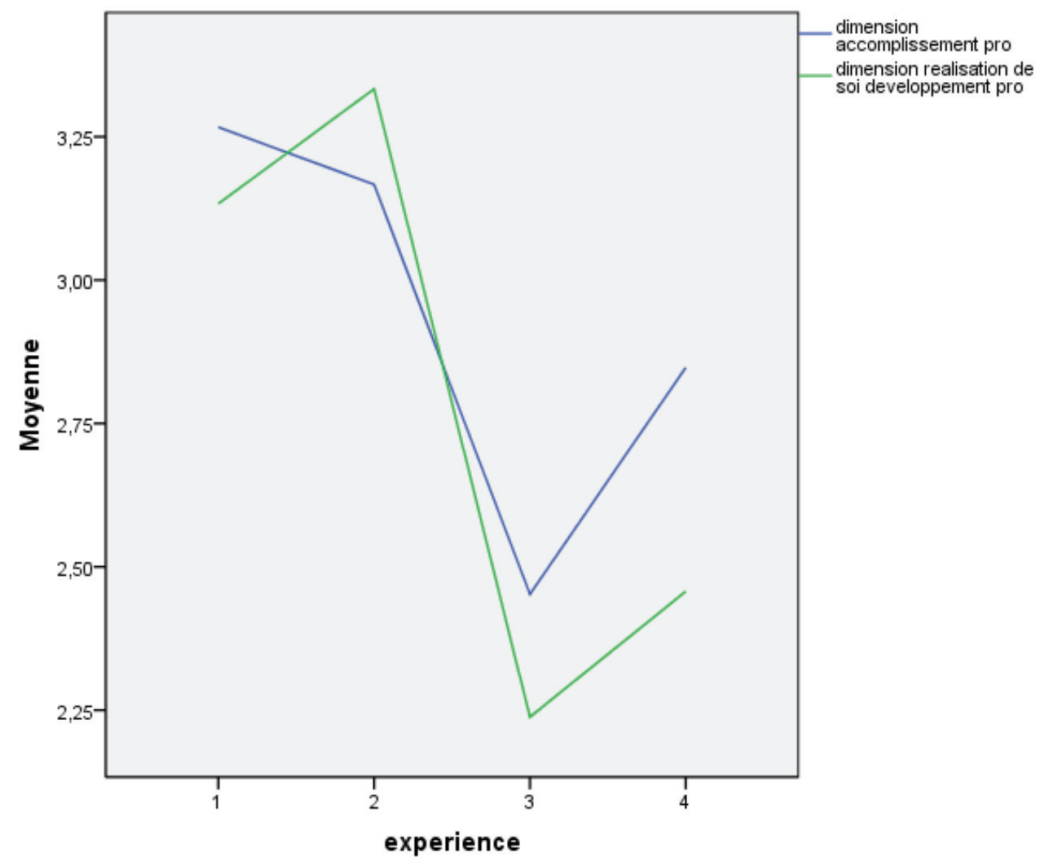

La figure 3 permet de conforter cette tendance pour la tranche d'âge de 25-45 ans et illustre une progression de la composante intrinsèque de la satisfaction professionnelle chez les enseignant.e.s dans la tranche d'âge des 46-60 ans.

\section{Figure 3}

Évolution des composantes intrinsèques et extrinsèques de la satisfaction professionnelle en fonction de la classe d'âge des enseignant.e.s

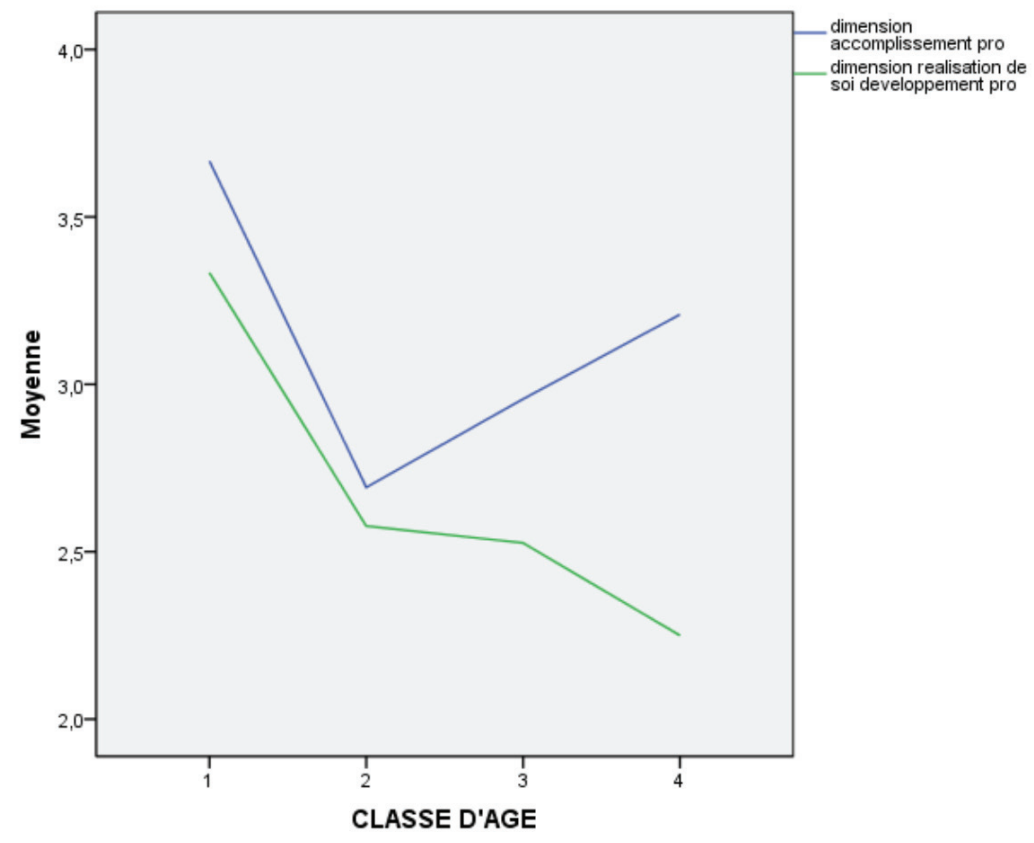


La satisfaction professionnelle des enseignant-e-s du point de vue de ses composantes extrinsèques et des variables de contrôle

Si l'on examine les rapports entre la réalisation de soi et le développement professionnel des enseignant·e.s avec leur sexe biologique, il apparait que les hommes considèrent que le métier a plus d'inconvénients que d'avantages (ST5; $F=5,102 ; p=.02$ ), qu'ils manquent de marges de liberté et de possibilités d'initiative (ST8 ; $F=6,709$; $p=.01$ ) et se sentent insuffisamment considérés par la communauté éducative (ST11; $F=4,610 ; p=.03$ ).

$\mathrm{Au}$ niveau global de notre échantillon, il apparait que les avantages et inconvénients du métier sont positivement et significativement corrélés avec les possibilités de formation proposées (ST12; $r=.43$; $p<0.001$ ), avec la reconnaissance effective de l'investissement et du mérite par l'employeur ou la direction d'établissement (ST7 ; $r=.65 ; p<0.001)$, l'aspect plus ou moins agréable du travail en soi (ST4; $r=.48 ; p<0.001)$ et la perception des possibilités d'évolution de carrière (ST10; $r=.38 ; p<0.001$ ).

Nous avons voulu connaître l'influence de l'établissement scolaire de travail sur la perception de satisfaction de toutes et tous les enseignant.e.s de notre échantillon. Le test de T Student permet de considérer une différence significative entre celles et ceux exerçant en collège et celles et ceux en lycée. En collège, l'ensemble des enseignant.e.s perçoit plus d'inconvénients que d'avantages du métier (ST5; $F=8,624 ; p=.005$ ), moins de reconnaissance de leurs efforts et de leur mérite par l'employeur ou la direction d'établissement (ST7; $F=10,09$; $p=.003)$ et une faible considération exprimée par la communauté éducative (ST11; $F=8,27 ; p=.006)$.

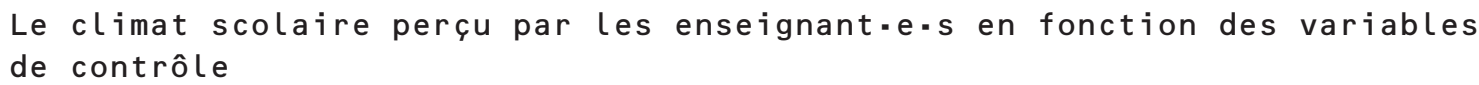

Les enseignant.e.s des deux établissements perçoivent le climat éducatif comme correct $\left(M_{\text {oyenne }}=3.05\right.$; $E T=.45)$ tout comme le climat de sécurité $\left(\mathrm{M}_{\text {oyenne }}=3.36 ; E T=.54\right)$. Le climat d'appartenance à une communauté scolaire $\left(\mathrm{M}_{\text {oyenne }}=2.84 ; E T=.62\right)$ et le climat pédagogique $\left(\mathrm{M}_{\text {oyenne }}=2.93 ; E T=.45\right)$ ont en revanche des moyennes plus faibles. Le climat d'appartenance à une communauté scolaire $\left(\mathrm{R}^{2}\right.$ ajusté $\left.=.44\right)$ correspond à l'indice global du phénomène étudié.

En ce qui concerne le climat de sécurité, le respect entre collègues (CEE10) est significativement et positivement corrélé avec la culture de coopération et de soutien mutuel perçue au sein de l'établissement (CEE3 ; $r=0.38 ; p<.001$ ), mais aussi avec le sentiment d'être soutenu.e par sa hiérarchie (CEE12; $r=0.40 ; p<.001$ ) et de ne pas être isolé.e par rapport aux difficultés de gestion des comportements (CEE16; $r=.40 ; p<.001$ ). La perception de se sentir en sécurité dans l'établissement est positivement et significativement corrélée avec l'absence d'une dégradation perçue du climat scolaire ces dernières années (CEE4; $r=0.36 ; p<.05$ ).

En ce qui concerne le climat pédagogique, le test de Mann-Whitney choisi en raison de l'absence d'homogénéité des variances $(p<.001)(F=3,864 ; p=.05)$ permet de rejeter l'hypothèse nulle d'égalité des variances entre les enseignant.e.s concerné.e.s par des arrêts maladie ces quatre dernières années et les autres. Le test de T Student permet d'affirmer une différence significative entre les deux groupes. Ainsi, les enseignant.e.s concerné.e.s par au moins un arrêt maladie au cours des quatre dernières années $\left(\mathrm{M}_{\text {oyenne }}=2.65 ; E T=.46\right)$ perçoivent un climat pédagogique plus dégradé que les autres $\left(\mathrm{M}_{\text {oyenne }}=3.17 ; E T=.33\right)$ soit $40 \%$ de notre échantillon et majoritairement les hommes.

Le climat d'appartenance à une communauté scolaire est significativement corrélé (CEE4) avec le sentiment d'être soutenu.e par la hiérarchie (CEE12; $r=.62 ; p<.001)$, l'existence d'un esprit collaboratif entre professionnel.le.s (CEE3 ; $r=.50 ; p<.001)$ et la perception de ne pas être isolé.e dans la gestion des problèmes (CEE16; $r=0.47 ; p<.001)$.

Ce facteur du climat scolaire ayant une faible moyenne au sein de notre échantillon, nous avons souhaité savoir si cette perception variait en fonction de l'établissement. Au regard de l'homogénéité des variances $(F=4,369$; $p=.042)$ entre les deux types d'établissements, nous avons réalisé le test de $T$ Student. Ce test confirme que les enseignant.e.s de collège ont une moyenne de perception du climat d'appartenance à une communauté scolaire $\left(M_{\text {oyenne }}=2,68 ; E T=.75\right)$ significativement plus faible que celle de leurs homologues en lycée $\left(M_{\text {oyenne }}=2,93\right.$; $E T=.52)$ (Figure 4). 
Figure 4

Climat d'appartenance à une communauté scolaire en fonction de l'établissement

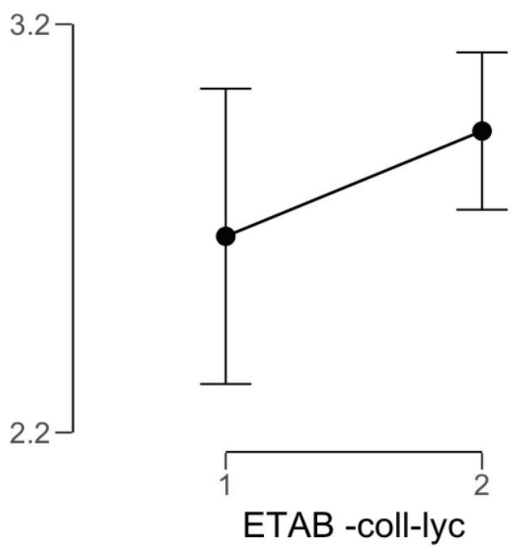

Analyse de régression hiérarchique entre la satisfaction professionnelle des enseignant-e.s et climat scolaire perçu

Nous souhaitions mesurer l'impact du climat scolaire perçu sur les composantes intrinsèques et extrinsèques de la satisfaction professionnelle $(r=.65 ; p<.001)$ (Tableau 6$)$. Tout d'abord, il apparait une corrélation positive et significative entre les composantes extrinsèques de la satisfaction professionnelle et le climat d'appartenance à une communauté scolaire $(r=0.49 ; p<.001)$, mais également avec le climat de sécurité et de respect perçu $(r=0.48 ; p<.001)$ et dans une moindre mesure avec le climat éducatif perçu $(r=0.40 ; p<.01)$.

La composante intrinsèque de la satisfaction professionnelle est quant à elle fortement et significativement corrélée avec le climat de cohésion et d'appartenance à une communauté scolaire $(r=0.65 ; p<.001)$ et dans une moindre mesure avec le climat éducatif $(r=0.34 ; p<.01)$ (Tableau 6). Autrement dit, plus la cohésion de la communauté scolaire est fragile et plus le sentiment d'accomplissement personnel des enseignant.e.s est affecté.

En ce qui concerne le climat de sécurité, indépendamment du sexe biologique et de l'établissement, celui-ci est perçu plus ou moins négativement en fonction de la tranche d'âge et des moyennes des composantes intrinsèques et extrinsèques de leur satisfaction professionnelle $(F 3,46=10.683 ; p<.001 ; t(46)=4.69>1.96 ; \mathrm{VIF}=$ $1.00<3 ; \mathrm{RMSE}=0.430 ; \mathrm{R}^{2}$ ajusté $=.41$ )

\section{Tableau 6}

Corrélations de Spearman entre les différents construits mesurés

\begin{tabular}{|c|c|c|c|c|c|c|c|}
\hline Facteurs ou composantes & & 1 & 2 & 3 & 4 & 5 & 6 \\
\hline \multirow{2}{*}{$\begin{array}{l}1 \text { Climat de cohésion } \\
\text { et d'appartenance à une } \\
\text { communauté scolaire }\end{array}$} & Spearman's rho & - & & & & & \\
\hline & $\mathrm{p}$-value & - & & & & & \\
\hline \multirow{2}{*}{2 Climat éducatif } & Spearman's rho & $0.324^{*}$ & 1 & & & & \\
\hline & $\mathrm{p}$-value & 0.011 & - & & & & \\
\hline \multirow{2}{*}{$\begin{array}{l}3 \text { Climat sécuritaire et de } \\
\text { respect }\end{array}$} & Spearman's rho & $0.399^{* *}$ & $0.245^{*}$ & 1 & & & \\
\hline & $\mathrm{p}$-value & 0.002 & 0.043 & - & & & \\
\hline \multirow{2}{*}{4 Climat pédagogique } & Spearman's rho & $0.325^{*}$ & $0.369^{* *}$ & $0.315^{*}$ & 1 & & \\
\hline & $\mathrm{p}$-value & 0.011 & 0.004 & 0.013 & - & & \\
\hline \multirow{2}{*}{5 Accomplissement personnel } & Spearman's rho & $0.659^{* * *}$ & $0.347^{* *}$ & $0.569^{* * *}$ & $0.401^{* *}$ & 1 & \\
\hline & $\mathrm{p}$-value & $<.001$ & 0.007 & $<.001$ & 0.002 & - & \\
\hline \multirow{2}{*}{$\begin{array}{l}6 \text { Réalisation de soi et } \\
\text { développement professionnel }\end{array}$} & Spearman's rho & $0.491^{* * *}$ & $0.403^{* *}$ & $0.480^{* * *}$ & $0.299^{*}$ & $0.504^{* * *}$ & 1 \\
\hline & $\mathrm{p}$-value & $<.001$ & 0.002 & $<.001$ & 0.018 & $<.001$ & - \\
\hline
\end{tabular}

Note $:{ }^{*} \mathrm{p}<.05,{ }^{* *} \mathrm{p}<.01,{ }^{* * *} \mathrm{p}<.001$, one-tailed 
Notre principal résultat en lien avec notre hypothèse de recherche est une relation significative de colinéarité qui existe à la fois entre toutes les composantes de la satisfaction professionnelle des enseignant.e.s et le climat de cohésion et d'appartenance à une communauté scolaire $(F 1.48=27.15 ; p<.001 ; t(48)=5.21>1.96$; $\mathrm{VIF}=1,00<3$; RMSE $=0.540 ; \mathrm{R}^{2}$ ajusté $\left.=.50\right)$ selon l'établissement scolaire de travail. La satisfaction professionnelle des enseignant.e.s est affectée par un manque de cohérence perçue dans les réponses collectives pour gérer les problèmes éducatifs, par des relations interpersonnelles dégradées entre enseignant.e.s et un mode de gouvernance de la direction d'établissement qui ne reconnaît pas suffisamment leur engagement dans le travail (Debarbieux et al., 2013).

\section{Discussion}

La qualité du vivre et travailler ensemble en milieu scolaire est essentielle pour que chacun puisse y apprendre, y développer des compétences et s'y réaliser pleinement en matière de bien-être et de santé au travail. Cette étude menée dans deux établissements proches géographiquement avait comme visée exploratoire d'examiner les rapports entre le climat scolaire perçu et la satisfaction professionnelle des enseignant.e.s.

La satisfaction professionnelle a été mesurée à l'aide de l'accomplissement personnel ( $\mathrm{R}^{2}$ ajusté $\left.=.46\right)$ d'une part et la réalisation de soi et le développement professionnel d'autre part $\left(\mathrm{R}^{2}\right.$ ajusté $\left.=.20\right)$. Le climat scolaire a été mesuré par quatre facteurs : le climat de cohésion et d'appartenance à une communauté scolaire $\left(\mathrm{R}^{2}\right.$ ajusté $\left.=.44\right)$, le climat de sécurité et de respect, le climat pédagogique et le climat éducatif.

Notre premier résultat concerne l'influence significative des variables de contrôle (sexe, âge, expérience et établissement d'affectation) sur les composantes intrinsèques et extrinsèques de la satisfaction professionnelle. Ce n'est qu'après dix ans d'expérience du métier que la moyenne d'accomplissement personnel augmente pour l'ensemble des enseignant.e.s de notre échantillon. Pour ce qui est de la réalisation de soi et du développement professionnel perçus, après la période de formation continuée obligatoire au cours des trois premières années d'exercice, ils ne cessent de décroitre jusqu'à 60 ans. Ce résultat va dans le même sens que ceux de l'étude de Fotinos et Horenstein (2011) sur le bien-être au travail du personnel des collèges et des lycées publics en France. Les auteurs soulignent ainsi que « $49 \%$ des enseignant[-e.]s estimaient que leur formation ne les avait pas préparé[-••.]s à leur travail actuel » et $40 \%$ « qu'ils [et elles] n’étaient pas formé[·e.]s ni soutenu[·.e.]s pour mettre en place des moyens pour améliorer leur travail » (p. 19), tout en déclarant dans $77 \%$ des cas souhaiter rester dans leur établissement. Par rapport à cela, les attentes des enseignant.e.s de notre échantillon vis-à-vis de la formation tout au long de la vie semblent importantes notamment pour des perspectives d'évolution de carrière. L'influence de l'âge sur la satisfaction professionnelle a été beaucoup étudiée. Notre résultat va dans le sens d'autres études où apparait une relation en forme de " $U$ " entre l'âge et la satisfaction professionnelle (Demirtas, 2010 ; Karsli et Iskender, 2009). Autrement dit, la satisfaction professionnelle décroit après les premières années au travail et augmente à nouveau à l'approche de la retraite. Dans le prolongement des résultats de ces études, nous identifions au sein de notre étude un profil d'enseignant.e.s insatisfait.e.s : $34.61 \%$ de notre échantillon composé d'hommes exerçant au sein du collège et dont l'âge est compris entre 25 et 45 ans avec moins de 10 ans d'expérience du métier. La composante intrinsèque de leur satisfaction professionnelle est significativement plus faible que chez les femmes. Ce résultat va dans le même sens que l'étude de Maroy (2008) menée auprès de la communauté française de Belgique dans laquelle les femmes enseignantes sont systématiquement plus satisfaites que les hommes, quels que soient les critères mis en avant. Comme le rappellent Florin et Guimard (2017) «dans toutes les études, quels que soient les domaines d'activité et le contexte de recherche » (p. 31), le sexe est un facteur explicatif de la satisfaction professionnelle comme le précise Afsa (2015) en prenant appui sur l'enquête TALIS (2013).

Notre second résultat porte sur les facteurs explicatifs de la satisfaction professionnelle en lien avec le climat scolaire perçu. Le climat de cohésion et d'appartenance à une communauté scolaire $\left(\mathrm{R}^{2}\right.$ ajusté $\left.=.35\right)$ et le climat de sécurité $\left(\mathrm{R}^{2}\right.$ ajusté $\left.=.34\right)$ influencent l'accomplissement personnel et suscitent l'insatisfaction professionnelle des enseignant.e.s ayant le profil explicité auparavant. Ce résultat rejoint ceux de l'étude menée par Sangsue et Vorpe (2004) sur le climat scolaire. Comme le rappellent Florin et Guimard (2017), le sentiment d'appartenance, qui renvoie aux dimensions groupales, est normalement un facteur de protection des atteintes à la santé au travail. Les enseignant.e.s insatisfait.e.s au sein de notre étude rencontrent des difficultés à travailler collectivement et à produire une réponse cohérente face aux problèmes éducatifs et scolaires des élèves. La satisfaction professionnelle des enseignant.e.s croît d'autant plus que des possibilités de soutien, d'entraide et de coopération leur sont proposées au sein de leur établissement scolaire. Il nous semble pertinent de retenir le fait 
que les enseignant.e.s du collège dans notre étude attendent de la reconnaissance et du soutien de la part de leur hiérarchie. Ce que la littérature du domaine nous permet d'affirmer, c'est que s'ils ou elles considèrent le mode de gouvernance de la direction d'établissement comme trop autoritaire et contrôlant, ils ou elles peuvent se désengager progressivement de leur travail et perdre encore en satisfaction professionnelle. En effet, la direction d'établissement est au centre d'une bonne cohésion d'équipe, de bonnes relations, notamment dans les relations école/famille. Elle participe de fait à ce que l'on nomme "l'effet établissement» (Florin et Guimard, 2017). Il s'agit donc ici d'une relation de pouvoir, au sens crozierien de contrôle des marges d'incertitude (Crozier et Friedberg, 1977) : des professionnel.le.s qui revendiquent une certaine autonomie pédagogique dans leur classe, et une direction d'établissement qui - au nom même du «travail en équipe " - est perçue comme trop contrôlante. Ceci pose largement la légitimité de ce pouvoir hiérarchique dans une organisation post-bureaucratique, mais encore largement pensée comme descendante (Dubet, 2016 ; Maroy, 2009).

\section{Conclusion}

Le climat scolaire est un concept complexe que nous avons choisi de traiter en le considérant comme un phénomène multidimensionnel et contextuel. Pour des préconisations adaptées aux établissements concernés par notre étude et à des fins d'un renforcement de la qualité du vivre ensemble, nous avons examiné la contribution de différents facteurs du climat scolaire sur la satisfaction professionnelle des enseignant.e.s (Lee et al., 2009). En matière de santé au travail, comme le résument Fleury-Bahi et al. (2009) «l'établissement scolaire doit être envisagé, à l'instar du quartier, de l'entreprise et du logement, comme un espace de vie qui peut contribuer au bien-être de l'individu ou, au contraire, générer de l'insatisfaction et du stress » (p. 201). Hubert (2015) suggérait dans son étude d'examiner le mode de gouvernance de la direction d'établissement dans la perception du climat scolaire par les élèves et par les personnels. Autrement dit, l'amélioration du climat scolaire perçu par les enseignant.e.s du collège passerait par ce que Debarbieux (2015) qualifie d'une « bienveillance vis-à-vis de [toutes les actrices et] tous les acteurs de l'école pour instaurer un bon climat scolaire " (p. 15). Il faut entendre par là une implication différente de la direction d'établissement dans la mesure où elle est le pivot du climat scolaire de l'établissement (Florin et Guimard, 2017) « au-delà d'être garant de la politique éducative de l'enseignement, secondaire, la direction est le représentant institutionnel, le référent» (p. 32). En matière de politiques éducatives en faveur d'une meilleure qualité de vie en milieu scolaire, la gouvernance constitue un facteur trop peu étudié de "l'effet établissement » et nous suggérons de le prendre en compte dans l'évaluation du bien-être perçu des enseignant.e.s. Dans le collège concerné par notre étude, l'insatisfaction professionnelle résulte moins de problèmes de gestion de comportements d'élèves que d'un manque de cohérence dans les réponses organisationnelles pour y faire face. Nous rappelons que le climat de cohésion et d'appartenance à une communauté scolaire renvoie au soutien de la direction, au mode de gouvernance et au travail avec l'équipe éducative (Payne et al., 2006). Afin de prévenir l'atteinte à la santé au travail des enseignant.e-s ayant un profil particulièrement insatisfait, nos recommandations sont ciblées sur la gouvernance de la direction d'établissement (Hubert, 2015 ; Weissbrodt et al., 2018). Nous suggérons d'explorer les modes participatifs et inclusifs de la gouvernance en faveur du bien-être des enseignant.e.s afin de mieux comprendre le rôle de ces formes de management dans la perception du climat scolaire et la qualité de vie en milieu scolaire. Comme le souligne Debarbieux (2015) :

L’enjeu serait celui d'une gouvernance renouvelée, qui se vivrait réellement moins comme un pilotage que comme un accompagnement, non seulement par le personnel enseignant, mais pour les différents cadres du système éducatif. Cela implique aussi que la créativité, voir le noble "bricolage " du terrain soit connu, reconnu et valorisé, non pas tant pour trouver la " bonne pratique à la mode » que pour mutualiser et casser la solitude des [actrices et] acteurs. (p. 24)

Sur le plan théorique, il serait également intéressant d'explorer le rôle de médiation tenu par le mode de gouvernance de la direction d'établissement dans les rapports entre la satisfaction professionnelle des enseignant.e.s et leur perception du climat scolaire notamment dans une perspective de santé au travail (Kyriacou, 2001 ; Sangsue et Vorpe, 2004 ; Skaalvik et Skaalvik, 2010). 


\section{Références bibliographiques}

Afsa, C. (2015). Où fait-il bon enseigner ? Éducation et Formations, 88-89, 61-77.

Amathieu, J., et Chaliès, S. (2014). Satisfaction professionnelle, formation et santé au travail des enseignants. Carrefours de l'éducation, 2(38), 211-238.

Barrère, A. (2002). Pourquoi les enseignants ne travaillent-ils pas en équipe ? Sociologie du travail, 44(4), 481-497. https://doi. org/10.1016/S0038-0296(02)01278-5

Beaudry, C., et Gagnon, M. (2019). L’obligation d'accommodement : une prise en charge teintée de la perception des acteurs. Revue d'études canadiennes, 53(1), 70-94. https://doi.org/10.3138/jcs.2018-0001

Benbenishty, R., Astor, R. A., et Astor, R. (2005). School violence in context : Culture, neighborhood, family, school, and gender. Oxford University Press.

Bentea, C.-C., et Anghelache, V. (2012). Teachers' motivation and satisfaction for professional activity. Procedia. Social and Behavioral Sciences, 33, 563-567. https://doi.org/10.1016/j.sbspro.2012.01.184

Brand, S., Felner, R. D., Seitsinger, A., Burns, A., et Bolton, N. (2008). A large scale study of the assessment of the social environment of middle and secondary schools: The validity and utility of teachers' ratings of school climate, cultural pluralism, and safety problems for understanding school effects and school improvement. Journal of School Psychology, 46(5), 507-535. https://doi.org/10.1016/j.jsp.2007.12.001

Ciavaldini-Cartaut, S., Marquié-Dubié, H., et d'Arripe-Longueville, F. (2017). Pénibilité au travail en milieu scolaire, stratégie de faire face et stratégie de défense chez les enseignants débutants : un autre regard sur les éléments contributifs d'une vulnérabilité au phénomène de décrochage professionnel. Perspectives interdisciplinaires sur le travail et la santé, 19(2). https://doi. org/10.4000/pistes.5099

Cohen, J., McCabe, E.-M., Michelli, N.-M., et Pickeral, T. (2009). School climate: Research, policy, teacher education and practice. Teachers College Record, 11(1), 180-213.

Crozier, M., et Friedberg, E. (1977). L'acteur et le système. Éditions du Seuil.

Debarbieux, E. (2015). Du " climat scolaire" : définitions, effets et politiques publiques. Education et Formation, 88-89, 11-27.

Debarbieux, E., et Fotinos, G. (2010). Violence et climat scolaire dans les établissements du second degré en France. Une enquête quantitative auprès des personnels de direction des lycées et collèges. Universite Victor Segalen Bordeaux, 2.

Debarbieux, E., Moignard, B., et Hamchaoui, K. (2013). Enquête de victimation et climat scolaire auprès des personnels de second degré. Ministère de l'Education Nationale, de la Jeunesse et du Sport. Répéréà https://www.academia.edu/11742893/ENQUE_ TE_DE_VICTIMATION_ET CLIMAT SCOLAIRE_AUPRE_S_DES_PERSONNELS_DU_SECOND_DEGRE

Demirtas, Z. (2010). Teachers' job satisfaction levels. Procedia Social and Behavioral Sciences, 9, 1069-1073. https://doi. org/10.1016/j.sbspro.2010.12.287

DeVellis, R. (2016). Développement d'échelle : théorie et applications (26). Sage Publications, Inc.

Dubet, F. (2016). Les instruments et l'institution : le cas de l'école. Sociologie du travail, 58(4), 381-389. https://doi.org/10.4000/ sdt. 807

Evers, W. J. G., Tomic, W., et Brouwers, A. (2004). Burnout among teachers: Students' and teachers'perceptions compared. School Psychology International, 25(2), 131-148. https://doi.org/10.1177/0143034304043670

Fleury-Bahi, G., Ndobo, A., Gardair, E., Jeoffrion, C., et Marcouyeux, A. (2009). Identification au lieu et aux pairs : quels effets sur la réussite scolaire? Cahiers Internationaux de Psychologie Sociale, 1(81), 97-113. https://doi.org/10.3917/cips.081.0097

Florin, A., et Guimard, P. (2017). La qualité de vie à l'école. Comment l'école peut-elle proposer un cadre de vie favorable à la réussite et au bien-être des élèves? CNESCO.

Fotinos, G., et Horenstein, J. M. (2011). La qualité de vie au travail dans les lycées et collèges : le burnout des enseignants. MGEN éditeur.

Gase, L. N., Gomez, L. M., Kuo, T. L., Glenn, B. A., Inkelas, M., et Ponce, N. A. (2017). Relationships among student, staff, and administrative measures of school climate and student health and academic outcomes. Journal of school health, 87(5), 319-328. https://doi.org/10.1111/josh.12501

Gottfredson, D. C. (2001). Delinquency and schools. Cambridge University.

Horenstein, J. M. (2006). La qualité de vie au travail des enseignants. État des lieux, analyse, propositions. MGEN éditeur.

Hubert, T. (2015). Le climat scolaire perçu par les collégiens. Education et Formations, 88-89, 80-101.

Janosz, M., Georges, P., et Parent, S. (1998). L'environnement socioéducatif à l'école secondaire : un modèle théorique pour guider l'évaluation du milieu. Revue Canadienne de Psycho-éducation, 27(2), 285-306.

Karsli M.-D., et Iskender H. (2009). To examine the effect of the motivation provided by the administration on the job satisfaction of teachers and their institutional commitment. Procedia Social and Behavioral Sciences, 1(1), 2252-2257. https://doi. org/10.1016/j.sbspro.2009.01.396

Kyriacou, C. (2001). Teacher stress: Directions for future research. Educational Review, 53(1), 27-35. https://doi. org/10.1080/00131910120033628

Lee, M.-S. M., Lee, M.-B., Liao, S.-C., et Chiang F.-T. (2009). Relationship: between mental health and job satisfaction among employees in a medical department of laboratory medicine. Journal of the Formosan Medical Association, 108(2),146-154. https://doi.org/10.1016/S0929-6646(09)60045-0

Locke, E. A. (1976). The nature and causes of job satisfaction. In N. Dunette (Ed.), Handbook of industrial and organizational pshychology (pp. 1297-1349). Rand-McNally.

Maroy, C. (2002). L'enseignement secondaire et ses enseignants. Une enquête dans le réseau d'enseignement libre subventionné en Communauté française de Belgique. De Boeck/Larcier.

Maroy, C. (2008). Perte d'attractivité du métier et malaise enseignant. Recherche et formation, 57, 23-38. https://doi.org/10.4000/ rechercheformation. 810

Maroy, C. (2009). Régulation post-bureaucratique des systèmes d'enseignement et travail enseignant. In Mottier Lopez L. et Crahay M. (Eds.), Évaluations en tension (pp. 83-99). De Boeck Supérieur. https://doi.org/10.3917/dbu.motti.2009.01 
Mitchell, M.-M., Bradshaw, C. P., et Leaf, P. J. (2010). Student and teacher perceptions of school climate: a multilevel exploration of patterns of discrepancy. Journal of School Health, 80(6), 271-279. https://doi.org/10.1111/j.1746-1561.2010.00501.x

OCDE. (2013). Talis 2013 : Enquête internationale sur l'enseignement et l'apprentissage. Technical report. OCDE éditions.

Payne, A., Gottfredson, D., et Gottfredson, G. (2006). School predictors of the intensity of implementation of school-based prevention programs: results from a national study. Prevention Science, 7(2), 225-237. https://doi.org/10.1007/s11121-0060029-2

Pett, M. A., Lackey, N. R., et Sullivan, J. J. (2003). Making sense of factor analysis. The use of factor analysis for instrument development in health care research. Sage.

Rascle, N., et Bergugnat, L. (2016). Qualité de vie des enseignants en relation avec celle des élèves : revue de question, recommandations. Rapport sur la qualité de vie à l'école. CNESCO. Repéré à https://www.cnesco.fr/fr/qualite-de-vie-a-lecole/

Sangsue, J., et Vorpe, G. (2004). Influences professionnelles et personnelles du climat scolaire chez les enseignants et les élèves. Psychologie du travail et des organisations, 10(4), 341-354. https://doi.org/10.1016/j.pto.2004.10.004

Seligman, M., Steen, T. A, Park., N., et Petterson, C. (2005). Positive psychology progress: Empirical validation of interventions. American Psychologist, 60(5), 410-421. https://doi.org/10.1037/0003-066X.60.5.410

Skaalvik, E. M., et Skaalvik, S. (2009). Does school context matter ? Relations with teacher burnout and job satisfaction. Teaching and Teacher Education, 25(3), 518-524. https://doi.org/10.1016/j.tate.2008.12.006

Skaalvik, E. M., et Skaalvik, S. (2010). Teacher self-efficacy and teacher burnout: a study of relations. Teaching and Teacher Education, 26(4), 1059-1069. https://doi.org/10.1016/j.tate.2009.11.001

Tardif, M., et Lessard, C. (1999). Le travail enseignant au quotidien : expérience, interactions humaines et dilemmes professionnels. Presses Université Laval.

Travers, C. J., et Cooper, C.L. (1996). Teachers under pressure: Stress in the teaching profession. Routledge.

Waasdorp, T. E., Pas, E. T., O’Brennan, L. M., et Bradshaw, C. P. (2011). A multilevel perspective on the climate of bullying: Discrepancies among students, school staff, and parents. Journal of school violence, 10(2), 115-132. https://doi.org/10.1080/1 5388220.2010 .539164

Weissbrodt, R., Arial, M., Graff, M., Ben Jamia, T., Villaret D’Anna, C., et Giauque, D. (2018). Prévenir les risques psychosociaux : une étude des perceptions et des pratiques des employeurs. Relations industrielles, 73(1), 174-203. https://doi. org/10.7202/1044431ar

Weiss, H. M. (2002). Deconstructing job satisfaction: Separating evaluations, beliefs and affective experiences. Human Resource Management Review, 12(2), 173-194. https://doi.org/10.1016/S1053-4822(02)00045-1

Mots clés: Gouvernance d'établissement ; santé au travail ; enseignant·e.s; climat scolaire ; satisfaction professionnelle

\section{Schulklima und Arbeitszufriedenheit der Lehrerinnen und Lehrer: eine Herausforderung für die Qualität des «gemeinsamen Lebens und Arbeitens in der Schule".}

\section{Zusammenfassung}

Die Qualität des Zusammenlebens und -arbeitens im schulischen Umfeld ist ein bildungs- und arbeitsmedizinisches Thema für alle Akteure an der Schule. Das Ziel dieser, in Frankreich durchgeführten Untersuchung, war es, den Einfluss des Schulklimas auf die Arbeitszufriedenheit zu bestimmen. Es wurden Daten von 50 freiwillig-teilnehmenden Lehrerinnen und Lehrern gesammelt, die an einer regionalen Mittel- und Oberschule arbeiten. Die Ergebnisse unterstreichen den Einfluss des Klimas durch die Zugehörigkeit zu einer Schulgemeinschaft auf intrinsische und extrinsische Komponenten der Arbeitszufriedenheit. Mehrere andere Faktoren (Alter, Geschlecht, Erfahrung, Art der Einrichtung) sind bestimmend für das Profil eher unzufriedener Lehrer/ innen. Für diese Schule werden Empfehlungen für die Schulgovernance im Hinblick auf die Gesundheit am Arbeitsplatz gegeben.

Schlagworte: Schulklima; Arbeitszufriedenheit; Regierung des Schulleiters; Arbeitsschutz Lehrer 


\section{Clima scolastico e soddisfazione professionale degli insegnanti: una sfida per la qualità del "vivere e lavorare insieme a scuola".}

Riassunto

La qualità della vita e della collaborazione nell'ambiente scolastico è una questione educativa e di salute sul lavoro per tutti gli interessati. Lo scopo di questa ricerca condotta in Francia era di determinare l'influenza del clima scolastico sulla soddisfazione sul lavoro. I dati sono stati raccolti da 50 insegnanti volontari che lavorano in una scuola media e superiore regionale. I risultati evidenziano l'impatto del clima di appartenenza ad una comunità scolastica sulle componenti intrinseche ed estrinseche della soddisfazione sul lavoro. Diversi altri fattori (età, sesso, esperienza, tipo di istituzione) determinano un profilo di insegnanti piuttosto insoddisfatti. Per questa scuola vengono fatte raccomandazioni sulla governance del preside a supporto della salute sul lavoro.

Parole chive: Clima scolastico; soddisfazione professionale; governance del preside scolastico; salute sul lavoro; insegnanti

\section{School climate and teachers' job satisfaction: a challenge for the quality of «living and working at school together"}

\section{Summary}

The quality of living and working together in the school environment is an educational and occupational health issue for all school stakeholders. The purpose of this research conducted in France was to determine the influence of school climate on job satisfaction. Data was collected from 50 volunteer teachers working in a regional middle school and high school. The results highlight the impact of the climate of belonging to a school community on the intrinsic and extrinsic components of job satisfaction. Several other factors (age, gender, experience, type of institution) determine a profile of rather dissatisfied teachers. For this school, recommendations are made regarding the school manager's governance with a view to occupational health.

Keywords: School climate; job satisfaction; school manager mode of governance; occupation health; teachers

Solange Ciavaldini-Cartaut. Après des études en sciences de l'éducation et de la formation, elle est actuellement Maître de conférences HDR et co-directrice du département des sciences de l'éducation de l'Université Côte d'Azur. Ses travaux portent notamment sur la formation des enseignant·e.s, le développement de l'activité professionnelle et les enjeux de santé.

Université Côte d'Azur, INSPE de l'académie de Nice, 89 avenue George V, F-06046 Nice Cedex 1

E-Mail : solange.cartaut@univ-cotedazur.fr

Catherine Blaya. Après des études en sociologie de l'éducation, elle est actuellement Professeure des Universités en sciences de l'éducation et de la formation et directrice de l'INSPE de l'académie de Nice. Ses travaux portent notamment sur la violence scolaire, la cyber-violence, le climat et le décrochage scolaire.

Université Côte d'Azur, INSPE de l'académie de Nice, 89 avenue George V, F-06046 Nice Cedex 1.

E-Mail : catherine.blaya@univ-cotedazur.fr 